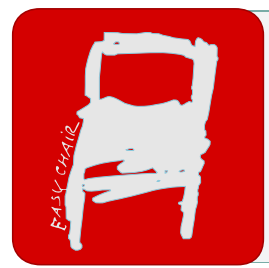

EPiC Series in Health Sciences

Volume 2, 2018, Pages 69-73

CAOS 2018. The 18th Annual Meeting of the International Society for Computer Assisted Orthopaedic Surgery

\title{
Robot assisted midline lumbar fusion with cortical bone trajectory screw
}

\author{
Da HE ${ }^{1}$, Qiang YUAN ${ }^{1}$, Lin $\mathrm{HU}^{1}$ and Wei TIAN ${ }^{1}$ \\ ${ }^{1}$ Department of Spine surgery, Beijing Jishuitan Hospital, Beijing, 100035, China \\ hedamd@vip.163.com, tianweijstevip.163.com
}

\begin{abstract}
Objective: Midline lumbar fusion with cortical bone trajectory(CBT) screw is a novel technique operation, robot system is the new developed system which can help CBT insertion. In this retrospective study, we compare the CBT accuracy by the assistance of navigation system and robot system. Clinical result is also compared.

Result: 55 patients were involved in this retrospective study, 29 patients are assisted by navigation system, 26 patients are by robot system. Mean follow-up is over 12 months. The mean VAS is significant improved at the final follow up for navigation group from $6.2 \pm 1.5$ to $3.2 \pm 1.1$ (back pain), $7.5 \pm 0.9$ to1.8 \pm 0.7 (leg pain), same as the robot system from $6.8 \pm 1.5$ to $2.9 \pm 0.8$ (back pain), $7.7 \pm 1.0$ to $1.6 \pm 0.8$ (leg pain). The JOA score pre-operation is $14.7 \pm 4.5$ and $14.5 \pm 4.1$ for navigation and robot system, at the final follow up, it is $24.3 \pm 4.2$ and $23.9 \pm 4.4$. the number of penetration cortex has no difference between 2 groups $(\mathrm{n}=0.363)$.

Conclusion: MIDLF with CBT screw is effective for the treatment of lumbar degenerative disease. Using navigation and robot system will help the insertion of CBT screw safety and accurate, robot can release the surgeon from part of aiming work.
\end{abstract}

\section{Introduction}

Although pedicle screw fixation is still the most common and reliable method for management of lumbar degenerative disease, there are several disadvantages, such an invasive nature of traditional placement, potential risk for screw pullout for osteoporotic patients $[1,2,3]$.

During the last Decade, minimal invasive procedure becomes the requirement both for surgeon and patients [4]. The midline lumbar fusion with cortical bone trajectory Cortical bone trajectory (CBT) screw is a new technique provides an alternative minimal invasive fixation technique for lumbar spine [5]. Another advantage is that it provides a novel method for osteoporotic patients, because it increases the cortical bone contact, enhance the pullout strength of screw.

However, CBT screw technique is more difficult than pedicle screw, because cortical bone is not as good handle feeling as cancellous bone, even may cause fracture of the pars [8]. At first, we use 
navigation technique to guide the CBT screw. Recently, Robot technique provide a much easier method to assist this procedure [6].

\section{Material and Method}

From 2015 to 2016, 55 patients were involved in this retrospective study. All the patients are lumbar degenerative disease, including lumbar spondylolisthesis, lumbar stenosis. 29 patients are assisted by 3D-fluroscopy real time navigation(Styker). 26 patients use the Robot assisted technique. (TianJi Robot $\AA$, co-designed and modified by Beijing Jishuitan Hospital and Beijing Tinavi company).

The sequence of MIDLF procedure was as follows: midline posterior approach, length depend on the level involved (1 level 5-6cm, 2 level $8-10 \mathrm{~cm}$ ), dissect the paravertebral muscle, making of screw trajectory, laminectomy, PLIF or TLIF for bony union, and CBT screw fixation.

For navigation group, first, we use 3D fluoroscopy to get the date, then using navigation system to guide the insert point and angle, $2 \mathrm{~mm}$ high-speed drill to make the initial hole, tap the hole, during this procedure, apply navigation to correct the direction several times. Finally, use the fluoroscopy to check the position.

For robot group, use the 3D fluoroscopy to get the date, design the screw position on robot system, then robot direct the insertion point, use the elecdrill to put the Kirschner wire into the bone, use fluoroscopy to verify the position, hollow drill enlarges the tunnel.

Pre and post-operative visual analogue scale (VAS) score for both back pain and radicular symptoms, Japanese orthopaedic Association Score(JOA) were measured and analyzed. Penetration of the cortex was measured according to the Gertzbein-Robbins classification (A: no cortical violation; B: cortical breach $<2 \mathrm{~mm}$; C: $\geq 2 \mathrm{~mm}$ to $<4 \mathrm{~mm}$; D: $\geq 4 \mathrm{~mm}$ to $<6 \mathrm{~mm}$; E: $\geq 6 \mathrm{~mm}$ ) [7].

\section{Result}

The general information for both group are shown in table 1 .

For both Groups, there are significant difference between pre and post-operative in JOA score. (Table 2)

For computer navigation group, 140/146 were placed in good position $(<2 \mathrm{~mm}$, GertzbeinRobbins category), 4 were in assessed as type C. For robot assisted navigation group, for robot assisted group, 133/136 were safely placed,2 were in type C. No difference between 2 groups. $(\mathrm{p}=0.363)$

Table 1 general information of patients

\begin{tabular}{cccccc}
\hline Group & N & Male & Female & Number of screw & Follow-up (month) \\
\hline Navigation & 29 & 11 & 18 & 146 & 13.5 \\
Robot & 26 & 9 & 17 & 136 & 12.1 \\
\hline
\end{tabular}

Table 2 clinical result at pre-operation and final follow-up 


\begin{tabular}{cccccc}
\hline \multirow{2}{*}{ Group } & Back pain & Back pain & Leg pain & Leg pain & JOA(pre) \\
& VAS(pre) & VAS(final) & VAS(pre) & VAS(final) & \\
\hline Navigation & $6.2 \pm 1.5$ & $3.2 \pm 1.1(\mathrm{p}=0.02)$ & $7.5 \pm 0.9$ & $1.8 \pm 0.7(\mathrm{P}=0.006)$ & $14.7 \pm 4.5$ \\
Robot & $6.8 \pm 1.5$ & $2.9 \pm 0.8(\mathrm{p}=0.01)$ & $7.7 \pm 1.0$ & $1.6 \pm 0.8(\mathrm{p}=0.008)$ & $14.5 \pm 4.1$ \\
\hline
\end{tabular}

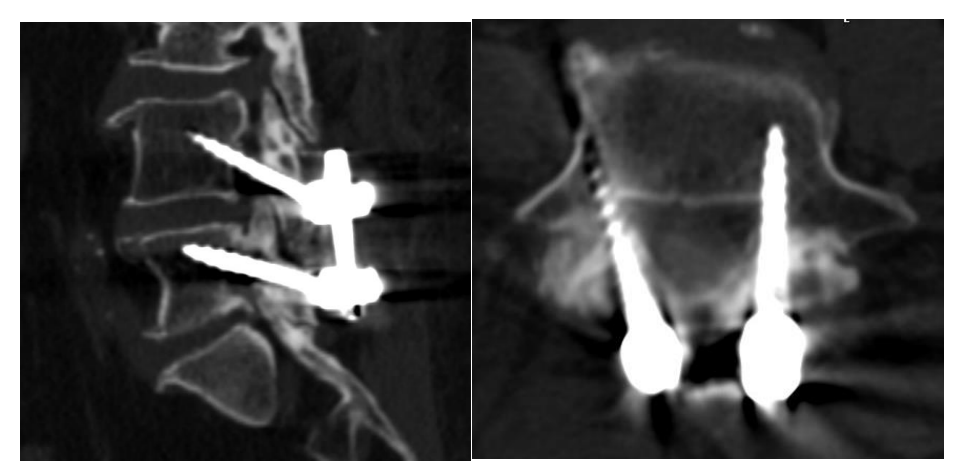

Figure 1: Postoperative computer tomography scans indicating the results of CBT position.

\section{Discussion}

CBT screw was first published by Santoni et al. in 2009[5]. The traditional PS approach to lumbosacral spine surgery requires extensive lateral spinal dissection for screw placement, however,the CBT procedure requires less soft tissue exposure, as screws are placed medially to laterally with a starting point at the junction between the lateral pars, it was thought as a minimal invasive operation. Biomechanical studies were shown that CBT screw pullout strength is superior to the traditional pedicle screw(PS), which make it as an alternative method for osteoporotic patients, however, clinical evidence is still limited to prove it $[9,10]$.

Ninomiya et al., and Takenaka et al. reported significant differences between preoperative and postoperative in back or leg pain among their subjects, but there is no difference between CBT and PS group $[11,12]$. However, Chen et al. described no difference in postoperative back pain immediately after surgery but noted that the CBT had a significantly higher VAS pain score at the final 8-month follow-up [13]. Chin et al. described no differences in postoperative back pain immediately after surgery but noted that the PS group had a significantly higher VAS Pain score at 2-year follow-up [14]. In our group, we didn't compare the PS with CBT screw, but both navigation group and robot group had a significant improvement in back pain and leg pain.

JOA score is a common used assessment system for lumbar degenerative disease. In the normal population, the total JOA score is a full 29 points. The JOA evaluates symptoms, functionality, and clinical signs of low-back pain and may be used in pre- and post-operative evaluation of patients. Mori et al. showed that mean post-operative and latest follow-up JOA scores were significantly increased from mean pre-operative scores in patients who underwent CBT [15]. Sakaura et al. investigated JOA scores in patients who underwent CBT, pre-operative and post-operative JOA scores were significantly improved when compared [16]. In both our group, we have get the same result in the final follow-up. 
Computer assisted technology is the best partner of the complex screw insertion procedure such as the minimal invasive surgery of spine, CBT screw, upper cervical screw, or secondary operation. Navigation is the past generation, robot is the newest system, which replace the part of hand function of surgeon. There are still no report of robot assisted CBT screw, in our study, both navigation and robot assisted system make the CBT screw insertion accurate, but robot system shows advantage of decrease the depending on doctors decision which make the procedure easier.

\section{Conclusion}

Our results indicate that MIDLF with CBT is effective for the treatment of lumbar degenerative disease. Using navigation and robot assisted procedure make the insertion of CBT screw safety, robot can replace part function of doctor.

\section{References}

1. Kaiser MG, Eck JC, Groff MW, et al. Guideline update for the performance of fusion procedures for degenerative disease of the lumbar spine. Part 1: introduction and methodology. $\mathrm{J}$ Neurosurg Spine 2014;21:2-6.

2. Wu AM, Zou F, Cao Y, et al. Lumbar spinal stenosis: an update on the epidemiology, diagnosis and treatment. AME Med J 2017;2:63 10.

3 Boucher HH. A method of spinal fusion. J Bone Joint Surg Br 1959;41:248-59.

4 Kim CW, Siemionow K, Anderson DG, Phillips FM: The current state of minimally invasive spine surgery. J Bone Joint Surg Am 93: 582- 596, 2011.

5 Santoni BG, Hynes RA, McGilvray KC, Rodriguez-Canessa G, Lyons AS, Henson MA, Womack WJ, Puttlitz CM: Cortical bone trajectory for lumbar pedicle screws. Spine J 9: 366- 373, 2009.

6 W. Tian, H. Wang, Y.J. Liu, Robot-assisted Anterior Odontoid Screw Fixation: A Case Report, Orthopaedic surgery 8(3) (2016) 400-4.

7 I. Pechlivanis, G. Kiriyanthan, M. Engelhardt, M. Scholz, S. Lucke, A. Harders, K.

Schmieder, Percutaneous placement of pedicle screws in the lumbar spine using a bone mounted miniature robotic system: first experiences and accuracy of screw placement, Spine 34(4) (2009) 3928.

8 Patel SS, Cheng WK, Danisa OA. Early complications after instrumentation of the lumbar spine using cortical bone trajectory technique. J Clin Neurosci. 2016 Feb;24:63-7.

9 Ninomiya K, Iwatsuki K, Ohnishi YI, Ohkawa T, Yoshimine T. Significance of the Pars Interarticularis in the Cortical Bone Trajectory Screw Technique: An In Vivo Insertional Torque Study. Asian Spine J. 2016 Oct;10(5):901-906.

10 Mai HT, Mitchell SM, Hashmi SZ, Jenkins TJ, Patel AA, Hsu WK. Differences in bone mineral density of fixation points between lumbar cortical and traditional pedicle screws. Spine J. $2016 \mathrm{Jul} ; 16(7): 835-41$.

11 Ninomiya K, Iwatsuki K, Ohnishi YI, et al. Radiological evaluation of the initial fixation between cortical bone trajectory and conventional pedicle screw technique for lumbar degenerative spondylolisthesis. Asian Spine J 2016;10:251-7.

12 Takenaka S, Mukai Y, Tateishi K, et al. Clinical outcomes after posterior lumbar interbody fusion: comparison of cortical bone trajectory and conventional pedicle screw insertion. Clin Spine Surg 2017. 
13 Chen YR, Deb S, Pham L, et al. Minimally invasive lumbar pedicle screw fixation using cortical bone trajectory-a prospective cohort study on postoperative pain outcomes. Cureus 2016;8:e714.

14. Chin KR, Pencle FJ, Coombs AV, et al. Clinical outcomes with midline cortical bone trajectory pedicle screws versus traditional pedicle screws in moving lumbar fusions from hospitals to outpatient surgery centers. Clin Spine Surg 2017;30:E791-7.

15 Mori K, Nishizawa K, Nakamura A, et al. Short-term clinical result of cortical bone trajectory technique for the treatment of degenerative lumbar spondylolisthesis with more than 1-year follow-up. Asian Spine J 2016;10:238-44.

16 Sakaura H, Miwa T, Yamashita T, et al. Posterior lumbar interbody fusion with cortical bone trajectory screw fixation versus posterior lumbar interbody fusion using traditional pedicle screw fixation for degenerative lumbar spondylolisthesis: a comparative study. J Neurosurg Spine 2016;25:591-5. 